\title{
Comments on: Lower Incidence of Postoperative Pulmonary Complications Following Robot-Assisted Minimally Invasive Esophagectomy for Esophageal Cancer: Propensity Score- Matched Comparison to Conventional Minimally Invasive Esophagectomy
}

\author{
Rupen Shah $^{1}$, and Zane Hammoud ${ }^{2}$ \\ ${ }^{1}$ Division of Surgical Oncology, Department of Surgery, Henry Ford Hospital, Detroit, MI; ${ }^{2}$ Department of Surgery, Henry \\ Ford Hospital, Detroit, MI
}

The article by Tsunoda et al. ${ }^{1}$ adds to the growing body of literature evaluating outcomes following robot-assisted esophagectomy. The authors compared outcomes of "conventional" minimally invasive esophagectomy (MIE) using a thoracoscopic approach with six ports to robot assisted minimally invasive esophagectomy (RAMIE) with five ports. They primarily compare 30-day/short-term postoperative outcomes with a focus on pulmonary complications. ${ }^{2}$ Specifically, the authors included pneumonia and atelectasis requiring bronchoscopy or antibiotics as the pulmonary complications evaluated/tracked. They perform propensity matching using nine variables in an attempt to match the two groups based on nine covariates, including age, sex, body mass index (BMI), American Society of Anesthesiologists (ASA) physical status classification, tumor location, clinical $\mathrm{T}$ stage, clinical $\mathrm{N}$ stage, clinical TNM stage, and preoperative therapy. The majority of the patients had squamous cell carcinoma (94\%), with approximately $60 \%$ of the tumors located in the lower onethird of the esophagus. When comparing the matched cohorts, they found that the overall postoperative morbidity rate for RAMIE was $51 \%$ in comparison with $73 \%$ for conventional MIE $(p=0.03)$ and the grade III or higher

(C) Society of Surgical Oncology 2020

First Received: 17 August 2020

Accepted: 19 August 2020;

Published Online: 29 September 2020

Z. Hammoud

e-mail: zhammou1@hfhs.org morbidity rates were lower in the RAMIE cohort (11\% vs. $29 \%, p=0.04)$. Pulmonary complications were significantly lower in the RAMIE group. They attribute the decrease in pulmonary complications to a lower incidence of recurrent laryngeal nerve (RLN) injury in the RAMIE cohort ( $7 \%$ vs. $20 \%, p=0.06$ ) and to the use of five ports for RAMIE instead of the six used in conventional MIE. RLN injury was evaluated using routine laryngoscopy on postoperative day 1 by an intensivist, and if there was a suspicion of RLN injury, the patient was referred to an otolaryngologist. RLN palsy was recorded based on the laryngoscopic evaluation in a dichotomous manner as absent or present. The severity of the RLN palsy, laterality, and its implications, i.e., aspiration, pneumonia, reintubation, and prolonged ICU stay and hospital stay are not presented in the current manuscript. They hypothesize that "precise dissection with tremor-less flex instruments" used in RAMIE probably contributed to the lower incidence of RLN injury. Given that the majority of patients underwent a three-field lymphadenectomy, it is possible that some of these injuries were related to the cervical mobilization and/ or lymph node dissection and not to the thoracic portion of the procedure.

Interestingly, the lower incidence of pulmonary complications was noted despite significantly longer operative times in the RAMIE cohort. This is contrary to other studies that have noted an increased risk for pulmonary complications with longer operative times $[2,3]$. Furthermore, the authors state that another possible explanation for the lower pulmonary complications in the RAMIE group may be due to the use of one fewer port. This contention is 
also questionable. Moreover, as the authors themselves report in the discussion, potential risk factors for postoperative RLN palsy, such as clinically large paratracheal nodes or bulky main tumor in the upper esophagus, were not adjusted for in this study.

One of the shortcomings of this study is the lack of data regarding the patient's preoperative pulmonary status, smoking history, or pulmonary function tests. Given that the majority of patients in this study had squamous cell carcinoma (94\%), this may be significant because smoking is one of the primary risk factors for development of esophageal squamous cell carcinoma in Japan., ${ }^{2,4}$

In a study by Law et al., nearly $30 \%$ of patients who did not have evidence for RLN injury by clinical examination or indirect laryngoscopy still developed respiratory complications. This suggests that factors other than vocal cord mobility likely play a role in a patient's likelihood for swallowing abnormalities, risk of aspiration, and development of pulmonary complications. ${ }^{3}$ Traditionally, McKeown three-field lymphadenectomy is associated with a higher incidence of RLN palsy in comparison with Ivor Lewis esophagectomy, and we congratulate the authors for an impressively low RLN injury rate (7\%) using the robotic platform without compromising oncologic outcomes, i.e., lymph node yield. In summary, despite the low RLN injury rates, the current article does not allow us to draw any conclusions regarding the value of the robotic platform in reducing pulmonary complications over conventional MIE. While we are admitted proponents of robotic esophagectomy, this manuscript cannot in and of itself be used to promote RAMIE. It does, however, add to the growing body of literature that supports RAMIE.

\section{REFERENCES}

1. Tsunoda SO, K, Hisamori S, Nishigori T, Okamura R,Maekawa H, Sakai Y. Lower incidence of postoperative pulmonary complications following robot-assisted minimally invasive esophagectomy for esophageal cancer: propensity score-matched comparison to conventional minimally invasive esophagectomy. Ann Surg Oncol. 2020. https://doi.org/10.1245/s10434-020-09081-6

2. Atkins BZ, D'Amico TA. Respiratory complications after esophagectomy. Thorac Surg Clin. 2006;16(1):35-48

3. Law S, Wong KH, Kwok KF, Chu KM, Wong J. Predictive factors for postoperative pulmonary complications and mortality after esophagectomy for cancer. Ann Surg. 2004;240(5):791-800.

4. Lin Y, Totsuka Y, He Y, et al. Epidemiology of esophageal cancer in Japan and China. J Epidemiol. 2013;23(4):233-42.

Publisher's Note Springer Nature remains neutral with regard to jurisdictional claims in published maps and institutional affiliations. 